\title{
Alterations in the serum proteome profile during the development of ovarian cancer
}

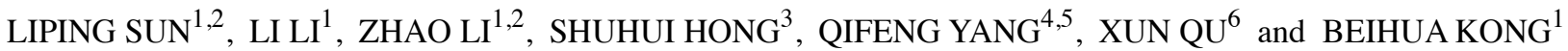 \\ ${ }^{1}$ Department of Obstetrics and Gynecology, Qilu Hospital, Shandong University, Jinan, Shandong 250012; \\ ${ }^{2}$ Medical College of Shandong University, Jinan, Shandong 250012; ${ }^{3}$ Department of Obstetrics and Gynecology, \\ Qianfoshan Hospital, Shandong University, Jinan, Shandong 250012, P.R. China; ${ }^{4}$ The Cancer Institute of New Jersey, \\ Robert Wood Johnson Medical School, New Brunswick, NJ 08903; ${ }^{5}$ Department of Genetics, Rutgers, \\ The State University of New Jersey, Piscataway, NJ 08854, USA; ${ }^{6}$ Department of Basic Medicine, \\ Shandong University, Jinan, Shandong 250012, P.R. China
}

Received July 27, 2014; Accepted September 11, 2014

DOI: $10.3892 /$ ijo.2014.2675

\begin{abstract}
Ovarian cancer causes more deaths than any other malignant tumor of the female reproductive system. This is because the condition usually goes undetected until the late stages. The purpose of the present study is to identify alterations in the serum proteome profile during the development of ovarian cancer and to provide an experimental basis for discovering new and valuable serum biomarkers for the early detection of ovarian carcinoma. Surface-enhanced laser desorption/ionization-time of flight (SELDI-TOF-MS) was used to profile changes in the serum proteome of Fischer 344 rats with ovarian cancer during the progress of tumor development. Sera were collected from the rats on day A (1 week before injection of tumor cells), day B (4 weeks after injection), and day $\mathrm{C}$ (6 weeks after injection). Each sample was subjected to SELDI-TOF-MS testing. Peak detection and alignment and selection of peaks with the highest discriminatory power were performed using ProteinChip biomarker software. Decision tree analyses were performed using biomarker pattern software. Finally, 3 peaks were found to be the most valuable ones (3759, 4659 and $9318 \mathrm{Da})$. The expression frequency of $\mathrm{m} / \mathrm{z}$ 3759-Da peaks was downregulated and another two frequencies (4659 and $9318 \mathrm{Da}$ ) were upregulated, and the levels of expression of these three proteins showed the same tendency as the expression frequency during the development of ovarian cancer. The total accuracy rate of diagnosis at 4 and 6 weeks post-injection was 94.7 and $97.3 \%$, respectively. Profiling the serum proteome changes during the process of the cancer
\end{abstract}

Correspondence to: $\mathrm{Dr} \mathrm{Li} \mathrm{Li}$, Department of Obstetrics and Gynecology, Qilu Hospital, Shandong University, 107 Wenhua Xi Road, Jinan, Shandong 250012, P.R. China

E-mail: 1ili0226@yeah.net

Key words: ovarian cancer, SELDI-TOF-MS, proteome, biomarker development using SELDI-TOF-MS may provide useful information regarding carcinogenesis and facilitate discovery of novel serum biomarkers for early detection.

\section{Introduction}

Ovarian cancer is the second most common gynecologic cancer and causes more deaths than any other malignant tumor of the female reproductive system (1). Unfortunately, the relatively asymptomatic nature of early ovarian cancer leaves most patients undiagnosed until advanced stages, which are usually incurable by surgery or chemotherapy. The majority of patients are diagnosed at an advanced stage, with a 5-year survival rate of only $35 \%$, compared to $90 \%$ for earlystage tumors, according to the International Federation of Gynecology and Obstetrics (FIGO) in 2013 (2-4). Identifying tumor biomarkers in biological fluids, such as serum, plasma and urine, is one of the most significant aspects of proteomic research. For this reason, it is necessary to understand the molecular alterations that occur during the development of ovarian cancer and use the information to more efficiently identify sensitive biomarkers suitable for the early detection of ovarian cancer.

Cancer cells secrete different kinds of proteins that can enter the blood circulation. Consequently, the serum proteome may reflect the abnormality or pathologic state of the disease (5). Comparative protein profiling is a promising way of detecting of specific protein expression of cancer, which may serve as a novel serum biomarker. Surface-enhanced laser desorption/ionization time-of-flight mass spectrometry (SELDI-TOF-MS) is a better tool for proteomic research than classic 2-dimensional electrophoresis. It combines chromatography with mass spectrometry, and it can provide rapid, reproducible, high-throughput protein profiles from trace biological samples $(6,7)$. Many studies have reported the use of SELDI-TOF-MS in the early diagnosis of cancers, such as lung cancer, esophageal cancer and breast cancer (8-11).

SELDI-TOF-MS was used to profile changes in the serum proteome changes of Fischer 344 rats with ovarian cancer 
during tumor development. The differential expression of proteinogram before and after tumor generation in rats offered useful information regarding proteins and genes that may be a key to carcinogenesis. It may also facilitate identification of novel serum biomarkers suitable for detection of ovarian cancer $(7,12)$.

\section{Materials and methods}

Cell lines and culture conditions. The Fischer 344-rat-derived epithelial ovarian carcinoma cell line NuTu-19 was a kind gift provided by Dr Airong Zhang of the Second Hospital of Shandong University. The NuTu-19 cells were maintained in complete media consisting of Dulbecco's modified Eagle's medium (DMEM; Gibco-Life Technologies) with 10\% heatinactivated fetal bovine serum (Gibco-Life Technologies) at $37^{\circ} \mathrm{C}, 5 \% \mathrm{CO}_{2}$ and $100 \%$ humidity.

The animal model. Female pathogen-free Fischer 344 rats (100-125 g) were obtained from Weitong Lihua, Inc., Beijing, China, and housed in a pathogen-free animal facility. Rats were kept in an environment with a 12-h light 12-h dark cycle and free access to food and water in the animal facility of the Shandong University Medical School. The present study was carried out in strict accordance with the guidelines for the Animal Care and Use of the Shandong University, China. The protocol was approved by the Committee on the Ethics of Animal Experiments in Qilu Hospital of Shandong University, and an informed consent form was provided by them. Surgery was performed under sodium pentobarbital anesthesia, and all efforts were made to minimize suffering.

NuTu-19 cells were harvested with $0.25 \%$ trypsin and $0.01 \%$ ethylenediaminetetraacetic acid (EDTA) and washed twice with phosphate-buffered saline (PBS) solution. Then a total of $10^{6}$ cells were injected intraperitoneally into each rat. The animals were observed daily and weighed weekly. All animals grew in parallel and the body weight of each rat was kept uniform throughout the experiment.

Serum sample preparation. Peripheral blood $(0.5 \mathrm{ml})$ was collected from the axillary vein of rats on day A (1 week before tumor cell injection), day B (4 weeks after injection), and day $\mathrm{C}$ (6 weeks following injection). Each blood sample was allowed to clot and was centrifuged at 3,000 rpm for 20 min soon after collection to remove all cellular components. Then, serum samples were aliquoted into $10-\mu 1$ sections and kept at $-80^{\circ} \mathrm{C}$ until use. The serum samples were centrifuged at $10,000 \mathrm{rpm}$ for $20 \mathrm{~min}$ at $4^{\circ} \mathrm{C}$ and then diluted $1: 3$ into $20 \mu \mathrm{l}$ U9 buffer solution (9 mol/1 urea, 2\% CHAPS, $50 \mathrm{mmol} / \mathrm{l}$ Tris-HCl, pH 9.0, 1\% DTT) before testing. After incubation on ice for $30 \mathrm{~min}$, the samples were diluted again 1:13 into $360 \mu \mathrm{l}$ of sodium acetate buffer solution (50 mmol/l NaAc, $\mathrm{pH} 4.0$ ). Then the samples were ready for SELDI testing.

SELDI analysis. Four different types of chip (Ciphergen Biosystems, Inc., Fremont, CA, USA) with surface chemistry of hydrophobic (H50), ionic (CM10), cationic (WCX2), and metal binding (IMAC3) were tested to determine which produced the best serum profiles. The CM10 ProteinChip, which is a weak cationic exchanger, was selected for the present study $(7,8)$. The CM10 ProteinChip was activated with $10 \mathrm{mM} \mathrm{HCl}$ and equilibrated with binding buffer $(100 \mathrm{mM}$ ammonium acetate). Then serum samples were added to the 8 -spot ProteinChip. The samples were allowed to incubate at room temperature for $60 \mathrm{~min}$ on a platform shaker, and then the array was washed twice with $200 \mu$ l binding buffer for $5 \mathrm{~min}$, followed by two quick rinses with HEPES water. Before SELDI analysis, $0.5 \mu 1$ saturated EAM solution (sinapinic acid in 50\% aqueous acetonitrile and $0.5 \%$ trifluoroacetic acid) was loaded onto each spot twice, allowing the surface of the array to air-dry between each EAM application $(8,13,14)$.

Chips loaded with samples were placed on the Protein Biological System II mass spectrometer reader (Ciphergen Biosystems). Time-of-flight spectra were generated by averaging 60 laser shots and collected in the positive mode at laser intensity 185 and detector sensitivity 8 with molecular weight optimized from 2,000 to 10,000 Da (15). Mass accuracy was calibrated on the day of measurements before data collection using the All-in-one peptide molecular mass standard (8).

The reproducibility of SELDI spectra from array to array on a single chip (intra-assay) and between chips (inter-assay) was determined using the pooled normal serum quality control (QC) sample (16). Three peaks in the range of 5,000-10,000 Da were selected randomly on spectra and used to calculate the coefficient of variance. The intra-assay analyses were performed in quadruplicate and the inter-assay analyses were performed on three different days.

Peak detection, data analysis and decision tree classification. Data analysis involved peak detection, alignment and selection of peaks with the highest discriminatory power. All spectra were collected using ProteinChip Biomarker software version 3.2 (Ciphergen Biosystems). Spectra range from 2,000 to $20,000 \mathrm{~m} / \mathrm{z}$ was selected for analysis. The study focused on this region to eliminate low-mass $(\mathrm{m} / \mathrm{z}<1,000)$ and low-intensity peaks $(\mathrm{m} / \mathrm{z}>20,000)$. Peak detection involved baseline subtraction, mass accuracy calibration and automatic peak detection (17). Mean spectra generated from different times were compared using the t-test.

Decision tree analyses were performed using Biomarker Patterns Software (BPS; Bio-Rad Laboratories). For each sample, the intensity values for each protein peak were entered in BPS and classified according to the tree analysis described. The BPS program can combine multiple biomarkers to distinguish between independent groups, thereby increasing sensitivity and specificity compared with single biomarker predictors. BPS was also used to perform a 10-fold crossvalidation because the size of the data set was too small for an independent validation set. This process allows the sensitivity and specificity to be predicted for future data and provides an accurate estimate of the predictive accuracy of the selected decision tree $(7,13,15)$.

\section{Results}

Tumor model. NuTu-19 cells grew progressively in the abdominal cavity in a manner typical of human ovarian epithelial carcinomas. At the fourth week after injection of $10^{6}$ cells, the 


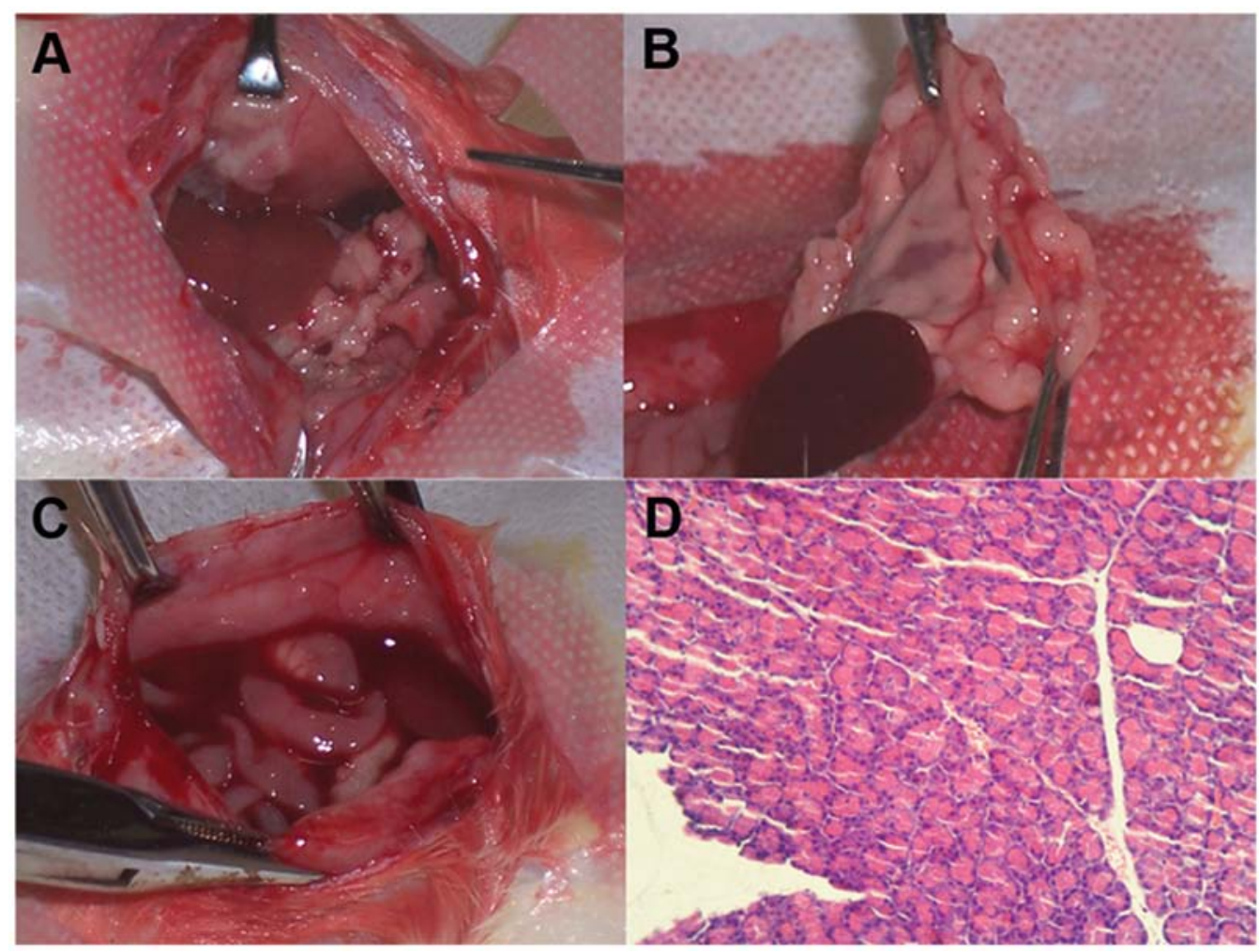

Figure 1. Tumor model. (A-C) At the 6th week after NuTu-19 cell injection, animals developed cancer in the peritoneal cavity, here represented by numerous serosal nodules (peritoneum, omentum, diaphragm and bowel), omentum contraction and malignant bloody ascites. (D) Pathohistological results: adenocarcinoma tissue with H\&E staining.

rats showed no obvious features of cancer. However, during the sixth week, all animals developed cancer in the peritoneal cavity, represented by numerous serosal nodules (peritoneum, omentum, diaphragm and bowel), omentum contraction and malignant bloody ascites. Characteristics of cachexia such as pallor (anemia), marasmus and abundance of bloody ascites appeared gradually. At the end of the study, all animals were sacrificed and tumor tissues were removed for section and H\&E staining. Pathohistological results confirmed the existence of adenocarcinoma tissue (Fig. 1).

Reproducibility. Accurate and reproducible feature selection is essential for SELDI application. The reproducibility of the current SELDI spectra was confirmed successfully, using a QC sample. The intra and inter-assay coefficients of variance for peak location were both $0.05 \%$, and the intra- and interassay coefficients of variance for normalized intensity were 12 and 18\%, respectively. Little variation was shown across day-to-day sampling and differences in instrumentation and chips $(16,18)$.

SELDI spectra of rat serum. SELDI spectra of rat serum samples showed a total of 126 raw peaks in the $\mathrm{m} / \mathrm{z}$ region of 1,000-20,000 Da. Using biomarker pattern software, the spectrum generated from pre-injection rats (1 week before injection) was compared to the spectrum generated from post-injection rats (4 and 6 weeks after injection, respectively). This comparison yielded a model consisting of 10 peaks that discriminated between pre-injection and post-injection rats during the development of ovarian cancer $(\mathrm{P}<0.05)(19)$.
Among these 10 peaks, the 3 peaks that were found to be most valuable after comprehensive selection had peak intensity levels above 5 . These 3 peaks corresponded to $\mathrm{m} / \mathrm{z}$ ratios of 3759, 4659 and 9318 (Fig. 2) (8,19).

Expression frequency of key proteins. The expression frequency of $\mathrm{m} / \mathrm{z} 3759$ peaks was downregulated and that of the other two peaks (4659 and 9318) was upregulated during the development of ovarian cancer. The protein with molecular weight 3759 Da was expressed in $86.8 \%$ of the rats before injection of ovarian cancer cells. However, 4 weeks after injection, the frequency dropped to only $35.2 \%$. At 6 weeks after injection, it had dropped to $8.4 \%$. None of the proteins of 4659 Da were detected before injection but levels increased to 2.7 and $39.0 \%$ at 4 and 6 weeks, respectively. Similarly, the expression rates of the 9318-Da protein were $31.6 \%$ before injection and 67.6 and $75.0 \%$ at 4 and 6 weeks (Fig. 3A) (20-22).

Expression levels of key proteins. The expression levels of the 3759-, 4659- and 9318-Da proteins, here indicated by the peak intensity of each protein, showed the same tendency as the expression frequency before and after injection. The peak intensity of the 3759-Da protein dropped from 19.19 \pm 5.2 before injection to $11.14 \pm 4.62(\mathrm{P}=0.0177)$ and $7.01 \pm 3.65$ $(\mathrm{P}=0.0008)$ at 4 and 6 weeks after. The peak intensity of the 4659-Da protein was $7.73 \pm 4.52$ before injection and increased gradually after tumor injection to $8.78 \pm 4.21(\mathrm{P}>0.05)$ at 4 weeks and $21.37 \pm 6.19(P=0.0014)$ at 6 weeks. Similarly, the peak intensity of protein 9318 Da before injection was 

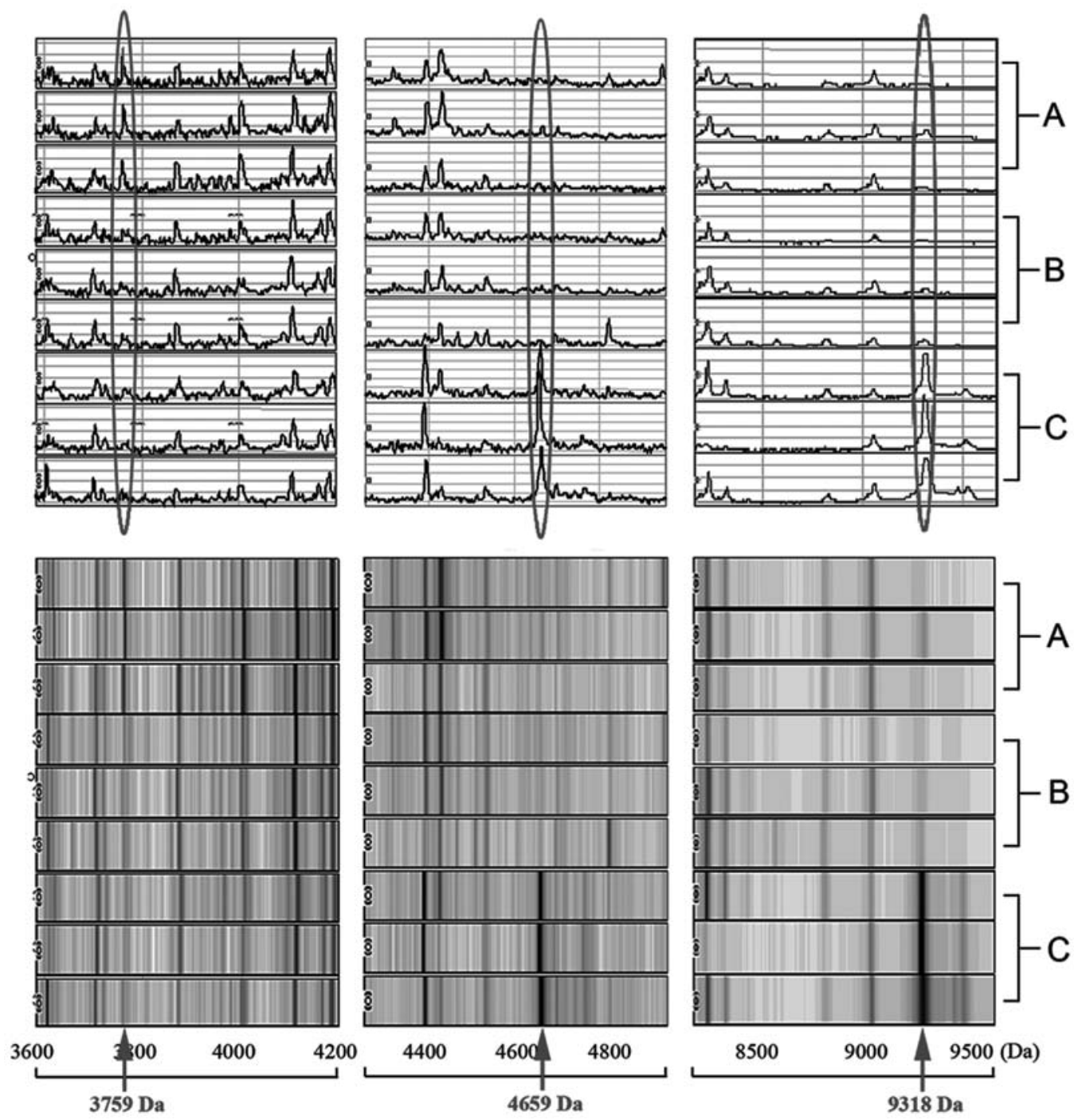

Figure 2. SELDI spectra of rat serum. Using biomarker pattern software, the spectrum generated from (A) rats 1 week before injection, (B) rats 4 weeks after injection and (C) rats 6 weeks after injection. These comparisons and comprehensive selection showed 3 peaks to be the most valuable. All had peak intensity above 5 . These 3 peaks corresponded to $\mathrm{m} / \mathrm{z}$ ratios of 3759,4659 and 9318 .

Table I. Diagnostic accuracy of tumor-bearing rats using diagnostic decision tree.

\begin{tabular}{lcccc}
\hline & & \multicolumn{3}{c}{ Diagnosis accuracy rate (\%) } \\
\cline { 3 - 5 } & $\mathrm{N}$ & Pre-injection & 4 weeks post-injection & 6 weeks post-injection \\
\hline Pre-injection & 38 & 86.84 & 5.26 & 7.89 \\
4 weeks post-injection & 38 & 5.26 & 76.30 & 18.42 \\
6 weeks post-injection & 37 & 2.70 & 16.22 & 81.08 \\
\hline
\end{tabular}

$1.5 \pm 1.03$, which was rather low, and subsequently increased to $3.01 \pm 4.07(\mathrm{P}>0.05)$ at 4 weeks and $7.67 \pm 4.99(\mathrm{P}=0.0141)$ at 6 weeks after tumor injection (Fig. 3B).

Establishment of a diagnostic decision tree. The decision classification tree was used as a predictive tool to distinguish the pre-injection rats from post-injection groups (23). The classification tree used 6 splitters with distinct masses of 4427.86, 9309.30, 5883.54, 4390.31, 5645.01 and 8219.00 Da, respectively. Cases were classified into 7 terminal nodes (Fig. 4). The error rate of the classification tree was estimated using cross-validation (24). At 4 and 6 weeks post-injection, the algorithm diagnosed $76.30 \%$ (29 of 38) and $81.08 \%$ (30 of 37) of cancer-bearing rats correctly and $86.84 \%$ (33 of 38) of the pre-injection rats were also diagnosed correctly. The total diagnostic accuracy rate of cancer-bearing rats at 4 and 6 weeks after injection were 94.7 and $97.3 \%$, respectively, in the present study (Table I). 

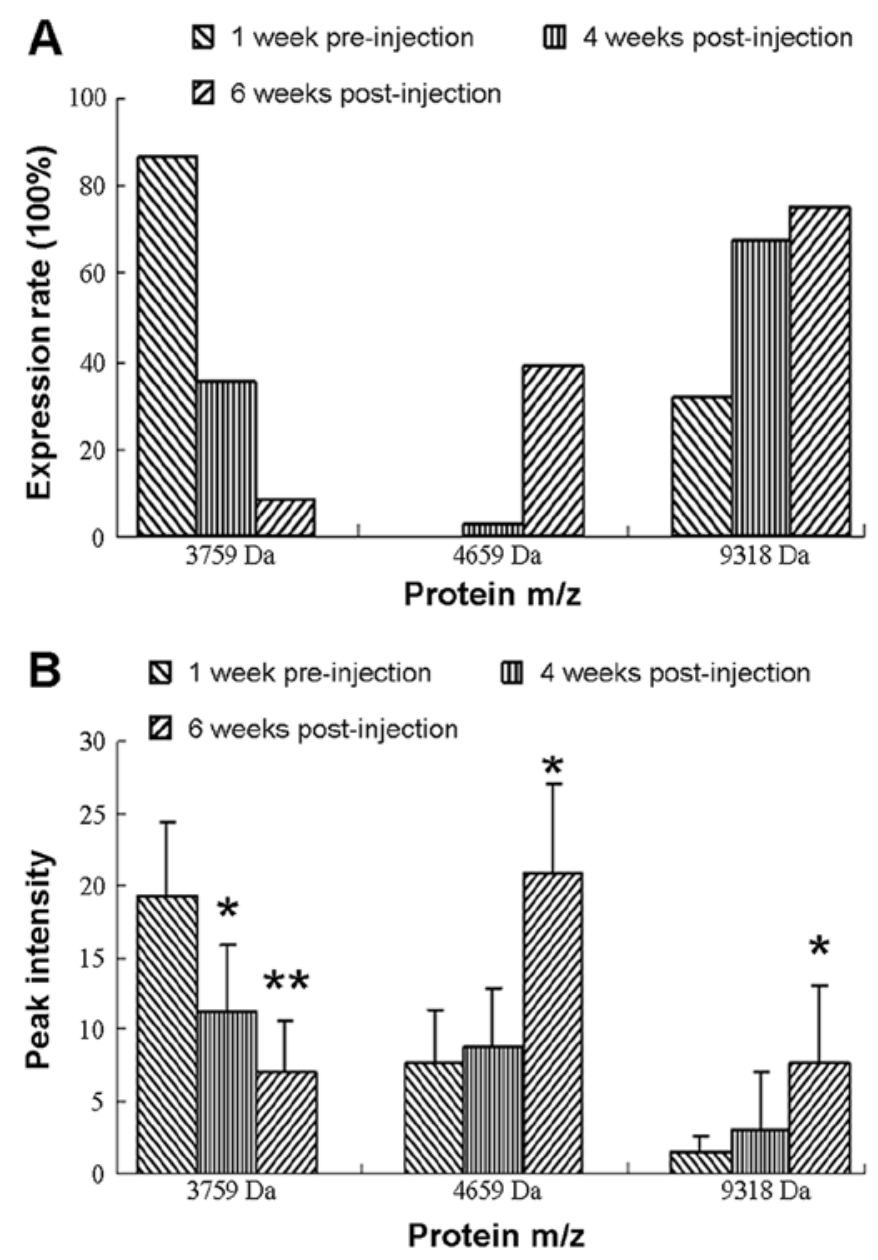

Figure 3. (A) Expression frequency/rate of key protein detection $(3759,4659$ and $9318 \mathrm{Da}$ ). The 3759 -Da protein was expressed in $86.8 \%$ of the rats before ovarian cancer cell injection, and the expression frequency dropped to only $35.2 \%$ at 4 weeks after injection and to $8.4 \%$ at 6 weeks. No 4659 -Da proteins were detected before injection but it was found in 2.7 and $39.0 \%$ of rats at 4 and 6 weeks after injection, respectively. The expression rate of the 9318-Da protein was $31.6 \%$ before injection and 67.6 and $75.0 \%$ at 4 and 6 weeks after injection, respectively. (B) Peak intensity of key proteins (3759, 4659 and $9318 \mathrm{Da}$ ). The peak intensity of 3759-Da protein dropped from $19.19 \pm 5.2$ before injection to $11.14 \pm 4.62\left({ }^{*} \mathrm{P}<0.05\right)$ and $7.01 \pm 3.65\left({ }^{* * *} \mathrm{P}<0.001\right)$ at 4 and 6 weeks after injection. The peak intensity of 4659 -Da protein was $7.73 \pm 4.52$ before injection and it increased gradually after injection to $8.78 \pm 4.21$ $(\mathrm{P}>0.05)$ at 4 weeks and $21.37 \pm 6.19\left({ }^{*} \mathrm{P}<0.05\right)$ at 6 weeks. The peak intensity of the 9318-Da protein before injection was $1.5 \pm 1.03$, and it increased to $3.01 \pm 4.07(\mathrm{P}>0.05)$ at 4 weeks and $7.67 \pm 4.99\left({ }^{*} \mathrm{P}<0.05\right)$ at 6 weeks after tumor cells injection. $\mathrm{N}=6$ for all experiments. Data are expressed as mean $\pm \mathrm{SD}$. $\mathrm{P}$ vs. 1 week pre-injection.

\section{Discussion}

For most types of cancers, survival rates depend on early diagnosis of the disease. For example, the 5-year survival rate of patients diagnosed at an advanced stage is $\sim 35 \%$, compared with $90 \%$ for cancers at stage I (2-4). According to the newest National Comprehensive Cancer Network (NCCN) guidelines for ovarian cancer, the diagnosis of ovarian cancer still depends mainly on imaging techniques and serum tumor markers testing. Current evidence shows that ultrasound imaging, CA-125, and human epididymis protein 4 (HE4) are useful in cancer diagnosis, but several of the markers used in recent studies, including CA-125, HE, B7-H4, mesothelin,

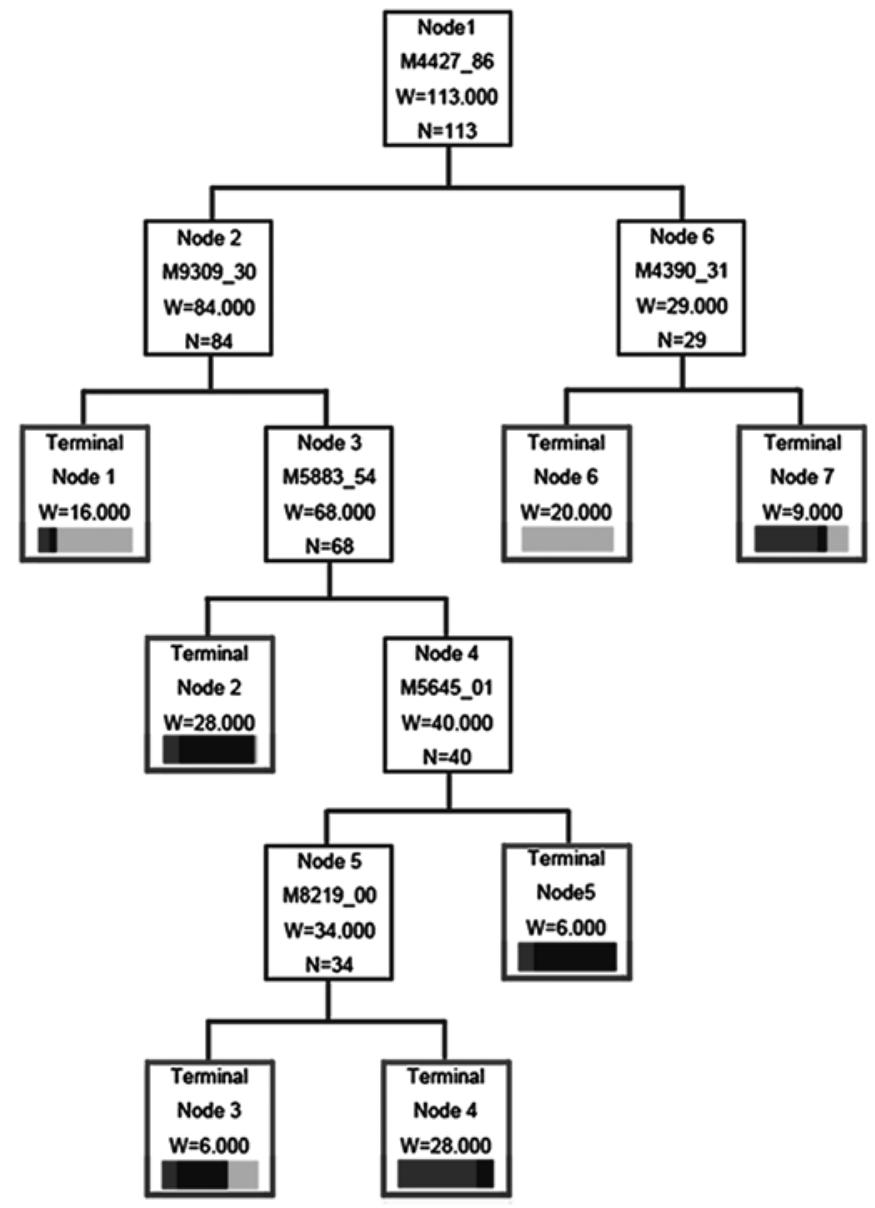

Figure 4. Diagnostic decision tree. Diagnostic decision tree used 6 splitters with distinct masses of 4427.86, 9309.30, 5883.54, 4390.31, 5645.01 and 8219.00 Da, respectively, and cases were classified into 7 terminal nodes.

decoy receptor 3 (DcR3) and spondin-2, are not adequately sensitive for use in early detection (25). However, the pathophysiological mechanisms of cancer development are not yet clear. Proteins carry out most cellular functions. The measurement of protein concentrations and activity levels can facilitate understanding of cancer pathogenesis. Once their roles in biology and pathology are understood, they may be used in the early detection of cancer. Traditional proteomics profiling techniques involve 2D gel electrophoresis, which is a lowthroughput approach to proteomic analysis. Surface-enhanced laser desorption/ionization time-of-flight mass spectrometry (SELDI-TOF-MS) technology, which is high-throughput, may also serve as an alternative to the 2D PAGE approach (26). SELDI-TOF-MS may help researchers better understand cellular functions at the protein level.

Mass spectrometry based on proteomics has been widely used in many related studies to identify and map proteins in bodily fluids, and proteomics profiling is one commonly used approach in proteomics (27). In recent years proteomics profiling using SELDI-TOF-MS has shown considerable potential in the field of biomarker discovery (28). Changes in proteomic profiles that occur around the time of tumorigenesis can facilitate understanding of the mechanism of cancer development. However, most of these experiments have involved non-clinical research and it is difficult to obtain proteomic 
profiles of patients before their diseases are diagnosed (29). In a previous study, serum samples from ovarian cancer rats and non-cancer controls were obtained using SELDI-TOF-MS. A four-peak model was established in the training set. It was found capable of distinguishing cancerous from non-cancerous samples with a sensitivity of $90.8 \%$ and specificity of $93.5 \%$. In a blind test of the same model, a sensitivity of $87.0 \%$ and a specificity of $95.0 \%$ were recorded (20). The purpose of the present study was to explore the changes in the proteomics profiles before and after tumor development using a Fischer 344 rat ovarian cancer model. As anticipated, key proteins were found during the development of ovarian cancer. A total of 126 raw peaks were found in the $\mathrm{m} / \mathrm{z}$ region of 1000-20,000 Da. A model consisting of 10 peaks was established to distinguish between pre-injection and post-injection rats. Of these 10 peaks, 3 peaks (m/z ratios of 3759,4659 and 9318 ) were found to be the relevant. The expression frequency of $\mathrm{m} / \mathrm{z} 3759$ peaks was downregulated and that of the other two peaks (4659 and 9318) was upregulated. This indicated that the first is a tumor suppressing gene and the other two are oncogenes. Any of these 3 proteins may serve as molecular targets in cancer therapy. They may also play key roles during tumorigenesis. Through establishing and analyzing a decision classification tree, we found the total diagnostic accuracy rate was also high enough (94.7\% at 4 weeks and $97.3 \%$ at 6 weeks). Studies have pointed out that the act of identifying these protein peaks is significant in some way. These protein signatures may facilitate the identification of populations at high risk of cancer and monitoring of patients' response to chemotherapy (30).

The present study evaluated the alterations in the proteomic profiling of the serum of tumor-bearing rats using SELDITOF-MS. These protein markers may be novel oncogenes or tumor suppressing proteins and may provide useful information on tumor cell growth and metastasis, genes related to the tumor cell microenvironment and specific molecular targets $(31,32)$. It may be helpful in the diagnosis and monitoring of ovarian cancer and the development of individualized treatment. Further research is needed to identify relevant proteins and to confirm the current findings in humans. Hopefully, new cancer biomarkers in human bodily fluids can be used to trace cancer development through proteomic analysis, providing an opportunity to put forward cancer control measures, design novel drugs and optimize the use of molecularly targeted agents (31). Although SELDI-TOF-MS is not a current stateof-the-art proteomics technology, it is still a guide to human ovarian cancer early diagnostics. We will focus further on the identity of the proteins for their eventual functional validation on cancer biology relevance in our subsequent experiments.

\section{Acknowledgements}

This study was funded by research grants from the National Natural Science Foundation of China (nos. 81100403) and Foundation for Outstanding Young Scientist in Shandong Province of China (BS2013YY035).

\section{References}

1. Maheedhar K, Bhat RA, Malini R, et al: Diagnosis of ovarian cancer by Raman spectroscopy: a pilot study. Photomed Laser Surg 26: 83-90, 2008.
2. Wegdam W, Moerland PD, Meijer D, et al: A critical assessment of SELDI-TOF-MS for biomarker discovery in serum and tissue of patients with an ovarian mass. Proteome Sci 10: 45, 2012.

3. Tessitore A, Gaggiano A, Cicciarelli G, et al: Serum biomarkers identification by mass spectrometry in high-mortality tumors. Int J Proteomics 2013: 125858, 2013.

4. Kristjansdottir B, Levan K, Partheen K, Carlsohn E and Sundfeldt K: Potential tumor biomarkers identified in ovarian cyst fluid by quantitative proteomic analysis, iTRAQ. Clin Proteomics 10: 4, 2013.

5. Sarojini S, Tamir A, Lim H, et al: Early detection biomarkers for ovarian cancer. J Oncol 2012: 709049, 2012.

6. Kamai T, Tomosugi N, Abe H, Kaji Y, Oyama T and Yoshida K: Protein profiling of blood samples from patients with hereditary leiomyomatosis and renal cell cancer by surface-enhanced laser desorption/ionization time-of-flight mass spectrometry. Int J Mol Sci 13: 14518-14532, 2012.

7. Liu C: The application of SELDI-TOF-MS in clinical diagnosis of cancers. J Biomed Biotechnol 2011: 245821, 2011.

8. Kelly P, Paulin F, Lamont D, et al: Pre-treatment plasma proteomic markers associated with survival in oesophageal cancer. Br J Cancer 106: 955-961, 2012.

9. Simsek C, Sonmez O, Keyf AI, et al: Importance of serum SELDI-TOF-MS analysis in the diagnosis of early lung cancer. Asian Pac J Cancer Prev 14: 2037-2042, 2013.

10. Opstal-van Winden AW, Krop EJ, Karedal MH, et al: Searching for early breast cancer biomarkers by serum protein profiling of pre-diagnostic serum; a nested case-control study. BMC Cancer 11: 381, 2011.

11. Gast MC, van Dulken EJ, van Loenen TK, et al: Detection of breast cancer by surface-enhanced laser desorption/ionization time-of-flight mass spectrometry tissue and serum protein profiling. Int J Biol Markers 24: 130-141, 2009.

12. Seibert V, Ebert MP and Buschmann T: Advances in clinical cancer proteomics: SELDI-TOF-mass spectrometry and biomarker discovery. Brief Funct Genomic Proteomic 4: 16-26, 2005.

13. Giusti I, D'Ascenzo S and Dolo V: Microvesicles as potential ovarian cancer biomarkers. Biomed Res Int 2013: 703048, 2013.

14. Wiesner A: Detection of tumor markers with ProteinChip technology. Curr Pharm Biotechnol 5: 45-67, 2004.

15. Mu AK, Lim BK, Hashim OH and Shuib AS: Identification of $O$-glycosylated proteins that are aberrantly excreted in the urine of patients with early stage ovarian cancer. Int J Mol Sci 14: 7923-7931, 2013

16. Callesen AK, Mogensen O, Jensen AK, et al: Reproducibility of mass spectrometry based protein profiles for diagnosis of ovarian cancer across clinical studies: a systematic review. J Proteomics 75: 2758-2772, 2012.

17. Emanuele VA II, Panicker G, Gurbaxani BM, Lin JM and Unger ER: Sensitive and specific peak detection for SELDI-TOF mass spectrometry using a wavelet/neural-network based approach. PLoS One 7: e48103, 2012.

18. Diao L, Clarke CH, Coombes KR, et al: Reproducibility of SELDI spectra across time and laboratories. Cancer Inform 10: 45-64, 2011.

19. Wang J, Zhang X, Ge X, Guo H, Xiong G and Zhu Y: Proteomic studies of early-stage and advanced ovarian cancer patients. Gynecol Oncol 111: 111-119, 2008.

20. Zhang H, Kong B, Qu X, Jia L, Deng B and Yang Q: Biomarker discovery for ovarian cancer using SELDI-TOF-MS. Gynecol Oncol 102: 61-66, 2006

21. Zhang Z, Bast RC Jr, Yu Y, et al: Three biomarkers identified from serum proteomic analysis for the detection of early stage ovarian cancer. Cancer Res 64: 5882-5890, 2004.

22. Moshkovskii SA, Serebryakova MV, Kuteykin-Teplyakov KB, et al: Ovarian cancer marker of $11.7 \mathrm{kDa}$ detected by proteomics is a serum amyloid A1. Proteomics 5: 3790-3797, 2005.

23. Cao XL, Li H, Yu XL, et al: Predicting early intrahepatic recurrence of hepatocellular carcinoma after microwave ablation using SELDI-TOF proteomic signature. PLoS One 8: e82448, 2013.

24. Qian JY, Mou SH and Liu CB: SELDI-TOF MS combined with magnetic beads for detecting serum protein biomarkers and establishment of a boosting decision tree model for diagnosis of pancreatic cancer. Asian Pac J Cancer Prev 13: 1911-1915, 2012.

25. NCCN: NCCN Clinical Guidelines in Oncology: Ovarian Cacer Including Fallopian Tube Cancer and Primary Peritoneal Cancer (Vesion 1.2014). 2014

26. Vlasova MA, Moshkovskii SA, Safarova MP, Makarov OV and Archakov AI: Molecular diagnostics of ovarian cancer using proteome techniques. Biomed Khim 51: 367-383, 2005 (In Russian). 
27. Huijbers A, Velstra B, Dekker TJ, et al: Proteomic serum biomarkers and their potential application in cancer screening programs. Int J Mol Sci 11: 4175-4193, 2010.

28. De Bock M, de Seny D, Meuwis MA, et al: Challenges for biomarker discovery in body fluids using SELDI-TOF-MS. J Biomed Biotechnol 2010: 906082, 2010.

29. Cadron I, Van Gorp T, Amant F, et al: The use of laser microdissection and SELDI-TOF MS in ovarian cancer tissue to identify protein profiles. Anticancer Res 29: 1039-1045, 2009.

30. Luo J, Qian JH, Yu JK, Zheng S, Xie X and Lu WG: Discovery of altered protein profiles in epithelial ovarian carcinogenesis by SELDI mass spectrometry. Eur J Gynaecol Oncol 29: 233-238, 2008.
31. Toss A, De Matteis E, Rossi E, et al: Ovarian cancer: can proteomics give new insights for therapy and diagnosis? Int J Mol Sci 14: 8271-8290, 2013.

32. Sinha A, Ignatchenko V, Ignatchenko A, Mejia-Guerrero S and Kislinger T: In-depth proteomic analyses of ovarian cancer cell line exosomes reveals differential enrichment of functional categories compared to the NCI 60 proteome. Biochem Biophys Res Commun 445: 694-701, 2014. 